\title{
Microscopy and its focal switch
}

\author{
Stefan W Hell
}

Until not very long ago, it was widely accepted that lens-based (far-field) optical microscopes cannot visualize details much finer than about half the wavelength of light. The advent of viable physical concepts for overcoming the limiting role of diffraction in the early 1990 s set off a quest that has led to readily applicable and widely accessible fluorescence microscopes with nanoscale spatial resolution. Here I discuss the principles of these methods together with their differences in implementation and operation. Finally, I outline potential developments.

Most textbooks still assert that a light microscope cannot resolve objects that are closer than about a quarter of a micrometer. However, as this issue of Nature Methods highlights, fluorescence microscopy has clearly turned into nanoscopy. And, as with many other leaps in science, this has not been due to gradual improvements from persistent technological progress. Lens-based microscopy has toppled a barrier that was thought would stand forever: the diffraction barrier imposed by the wave nature of light (Fig. 1).

According to Émile Verdet ${ }^{1}$, Ernst Abbe $^{2}$ and Lord Rayleigh ${ }^{3}$, light of a wavelength $\lambda$ focused by a lens of numerical aperture $n \sin \alpha<1$ cannot discern objects any closer together than distance $d=\lambda /(2 n \sin \alpha)$, because diffraction fuses their images into a single blur. In the same way, light cannot be focused more sharply than to a spot of $d \approx 200 \mathrm{~nm}$ in diameter and $400-700 \mathrm{~nm}$ in axial length, which corresponds to the resolution limit of basically all farfield light microscopes in the twentieth century. The resolution issue began to change in the early 1990s when $4 \mathrm{Pi}$ microscopy ${ }^{4-7}$ and $\mathrm{I}^{5} \mathrm{M}$ (refs. 8,9 ) improved the axial resolution by up to sevenfold. However, because these methods perfected focusing alone, they left the diffraction barrier entirely in place.

But now, a few years later, 'diffraction-unlimited' resolution has become most evident, accessible and even simple. The interested bystander is left wondering: What knocked the barrier over? What are the current limits? And what will be the limits in the future? In view

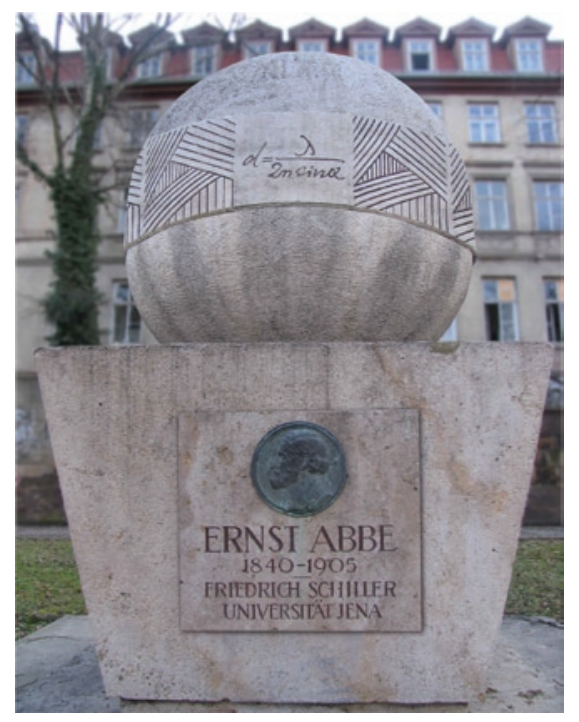

Figure 1 | Memorial erected in Ernst Abbe's honor displaying his equation describing the diffraction resolution limit, located in front of the physiology building of the University of Jena, Germany. (Courtesy of Rittweger, Sebesse MPI BPC)

of the fast pace that nanoscopy technology is taking, giving lasting answers is anything but prudent. Therefore, I have decided to leave the technical details aside and instead give a few insights into basic principles. After all, it is these principles that allow present methods to perform as they do and that determine what these methods will most likely achieve in the future.

Let us assume an unknown number of tiny fluorescent objects or molecules that are $D<200 \mathrm{~nm}$ apart. If they all possess different colors, separation is straightforward, as the right selection of filters will do the job. Discerning objects or molecules with distinct spectral characteristics has never been precluded by diffraction ${ }^{10}$. So if someone had figured out an effective way to label each little object in a sample with a different color, the recent developments would have been less essential. But what if there is no way to put differently colored stickers on them? The answer is to switch their signals on and off so 
Table 1 | Glossary of far-field fluorescence nanoscopy techniques

\begin{tabular}{ll}
\hline Technique & Description \\
\hline 4Pi microscopy & Improves the axial resolution in scanning far-field fluorescence three-dimensional \\
& microscopy by a factor of 3-7 using two opposing lenses of high numerical aperture. \\
& To sharpen the focal spot along the optical axis, the counterpropagating spherical \\
& wavefronts of the focused excitation light fields are coherently summed at the common \\
& focal point, and/or the spherical wavefronts of the emitted fluorescence light field \\
& are coherently summed at the detector. Depending on the specific implementation, \\
& mathematical deconvolution of raw data is either mandatory or optional. The concept \\
& essentially expands the aperture solid angle in microscopy. \\
\hline $\mathrm{I}^{5} \mathrm{M}$ & Improves the axial resolution in wide-field fluorescence three-dimensional microscopy. \\
& The scheme is similar to 4Pi microscopy. The counterpropagating excitation light field \\
& forms a flat standing wave, whereas emitted fluorescence light is collected through both \\
& lenses as spherical wavefronts that are summed at a common point of detection at a \\
& camera. Mathematical deconvolution of raw data is mandatory. It can also be combined \\
with lateral resolution improvement in a method referred to as 5 S S.
\end{tabular}

STED: stimulated emission depletion microscopy

A non-diffraction-limited form of scanning far-field fluorescence microscopy. Typically, fluorescence excitation created by a focused beam of excitation light is narrowed down in space by simultaneously applying a second spot of light for molecular de-excitation featuring a central zero (for example, a doughnut). The role of the de-excitation (STED) beam is to effectively confine molecules to the ground state, thus, effectively switching off the ability of the dye to fluoresce. De-excitation occurs within the nanosecond lifetime of the fluorescent state. Because no de-excitation occurs at the central zero, the excited state is established only in the region close to the zero.

GSD: ground state depletion microscopy

Analogous to STED microscopy. The area in which molecules can reside in the fluorescent

$13,30,31$ state is narrowed down in space by transiently switching the dyes to a metastable dark state-specifically, the triplet state. The use of a dark state of micro- to millisecond lifetime reduces the intensity required for the molecular switch in comparison to STED. The concept of switching fluorophores between long-lived bright and dark states has successively been extended to switching by cis-trans isomerization and other optically induced molecular bistabilities in the concept called RESOLFT.

(continued)

that they are seen consecutively. This is exactly how current far-field optical nanoscopy techniques resolve objects that are closer together than the diffraction limit. At the end of the day, the techniques known as STED ${ }^{11,12}, \mathrm{GSD}^{13}$, $\mathrm{SPEM}^{14}$ or $\mathrm{SSIM}^{15}$, RESOLFT ${ }^{16-19}$, PALM $^{20}$, STORM $^{21}$, FPALM $^{22}$, PAINT $^{23}$, dSTORM $^{24}$, TL-PALM ${ }^{25}$ and GSDIM $^{26}$ (Table 1) all discern objects closer than $200 \mathrm{~nm}$ by switching their fluorophores on and off sequentially in time.

\section{Switching characteristics}

To switch a fluorescent molecule on or off requires two states: a fluorescent (on) state and a dark (off) state, connected by a transition representing the actual switch. Several states in a fluorophore are suitable for such transitions ${ }^{16,18,27}$. The fluorescent singlet state $S_{1}$ and the ground state $S_{0}$ used in stimulated emission depletion (STED) microscopy are the most basic and obvious pair of bright and dark states. Excitation $\left(S_{0} \rightarrow S_{1}\right)$ makes the dye fluorescent, but applying a STED beam to quench it $\left(S_{1} \rightarrow S_{0}\right)$ switches the dye off. Two objects or molecules that would otherwise be blurred to a single spot can now be revealed individually by sequentially switching one of them off through STED. The same states are used in saturated pattern excitation microscopy (SPEM), also called saturated structured illumination microscopy (SSIM). Both ground state depletion (GSD) and ground state depletion-individual molecule return microscopy (GSDIM) pair the fluorescent singlet state with the longlived dark triplet state for the on-off switch. The concept referred to as reversible saturable optical fluorescent transition (RESOLFT) includes switching isomerization (cis-trans) states and other optically bistable transitions in fluorophores ${ }^{16-18}$, as do the landmark concepts photoactivation localization microscopy (PALM) and stochastic optical reconstruction microscopy (STORM). Because the number of states in a dye is limited, it is quite natural that several concepts rely on the same states and on similar mechanisms of fluorescence switching.

The shared molecular mechanism leads to common aspects in many of these concepts, and these can be readily understood by looking into the basics of an incoherently driven optical transition. If a molecule can be transferred from one state to the other by light, the probability that the molecule remains in the first state decreases exponentially with the beam intensity $I$ used; that is, it varies as $\exp \left(-I / I_{\mathrm{s}}\right)$. The 'saturation intensity' $I_{\mathrm{s}}$ is a characteristic of the transition used, scaling inversely with the lifetimes of the two states ${ }^{16,17}$. Applying intensities $I$ to a molecule that exceed $I_{\mathrm{s}}$ makes it $>63 \%$ probable that one of the photons brings about the switch; $I>5 I_{\mathrm{s}}$ makes it almost certain (>99\%). The longer the lifetime of the initial state, the more time we have to impinge upon it with a photon, and the longer the lifetime of the final state, the more durable the switch becomes. The lifetimes of the states can vary by orders of magnitude, and so does $I_{\mathrm{s}}$. The first dividing line between concepts is thus the switching mechanism and the intensities $I$ required.

For example, owing to its nanoseconds-long lifetime, switching off the fluorescent state $S_{1}$ by stimulated emission entails $I_{\mathrm{s}} \approx 5 \mathrm{MW} \mathrm{cm}{ }^{-2}$. Such intensities and higher are best achieved with spot scanning, which is why this implementation is usually preferred with STED ${ }^{16,17}$. Switching to the triplet or similar dark state of the fluorophores having lifetimes of micro- to milliseconds needs $10^{3}$ to $10^{6}$ times lower intensities ${ }^{13}$. Finally, $<10 \mathrm{~W} \mathrm{~cm}{ }^{-2}$ are required 
Table 1 | Glossary of far-field fluorescence nanoscopy techniques (continued)

\begin{tabular}{|c|c|c|}
\hline Technique & Description & References \\
\hline $\begin{array}{l}\text { SPEM/SSIM: saturated pattern excitation } \\
\text { microscopy or saturated structured } \\
\text { illumination microscopy }\end{array}$ & $\begin{array}{l}\text { A wide-field recording, highly parallelized scanning microscopy in which the molecules } \\
\text { are strongly excited to the fluorescent state, depleting the ground state (that is, switched } \\
\text { from the ground state to the fluorescent state) outside the line-shaped zeros produced by } \\
\text { a standing wave interference pattern. To cover the field of view, the pattern is scanned } \\
\text { across the specimen by phase-shifting the maxima of the interference pattern and reading } \\
\text { out the fluorescence imaged onto a camera for each scanning step. Because resolution is } \\
\text { improved only perpendicular to the line-shaped zeros, the pattern is tilted several times } \\
\text { to cover all directions in the focal plane. Mathematical analysis of the data renders super- } \\
\text { resolved images. Even scanning a single line-zero would give super resolution, but the } \\
\text { use of an array of lines parallelizes the process over a large area. }\end{array}$ & 14,15 \\
\hline
\end{tabular}

RESOLFT: reversible saturable/switchable optically linear fluorescence transition
A generalization of STED and GSD microscopy for molecular switching, including switching of reversibly activatable proteins and organic fluorophores. Switching can be regarded as a perfect saturable transition from one state to the other. The terminology 'saturated transition' is used in conjunction with molecular ensembles to also account for the fact that in an ensemble the population of the two states may equilibrate to fractions-say, $90 \%$ in the off state and $10 \%$ in the on state.

(F)PALM, STORM: (fluorescence) photoactivation localization microscopy, stochastic optical reconstruction microscopy while the molecules are in the on state. Calculating the centroid of the diffraction blob produced by each molecule and registering the coordinates of each molecule produces
Switches individual molecules stochastically and sparsely on by light-induced activation $20-22,36,41,42,49,79$ and then off, to detect a bunch of $m$ photons from a single molecule on a camera, emitted an image consisting of individual molecule positions. STORM has been initiated with pairs of photochromic cyanine dyes with one of them used as an activation (switch-on) facilitator.

\section{dSTORM: direct STORM} A simplified version of STORM that refrains from using a special dye for activation.

Switches off by depleting the molecular ground state and shelving the dye molecules in their triplet state, as in GSD. However, unlike GSD, it uses a stochastic readout, as in PALM, STORM, FPALM and dSTORM. It differs from these stochastic methods in that the dye molecule is not optically activated but is automatically switched on after its spontaneous return from the dark (triplet) state to its singlet state.

PAINT: point accumulation for imaging A method in which the 'on' state is generated by the binding of something to the structure 23 to be imaged, and the 'off' state by free diffusion or another dark (bleached) state.

GSDIM: ground state depletion followed by individual molecule return
$16-18,87,88$ to sharply define the coordinates where the molecule is on or off at any time point $t$. If the wavelength of $I(x, t)$ is selected to switch the dye off, applying $I(x, t)>5 I_{\mathrm{s}}$ everywhere outside the zero confines the 'on' state to the zero and its immediate proximity. Conversely, if $I(x, t)$ is chosen to switch the dye on, adjusting $I(x, t)>5 I_{\mathrm{s}}$ outside the zero confines the 'off' state in the same way. Surrounding the zero point with peak intensities $I_{\max }>>I_{\mathrm{s}}$ confines one of the states to a narrow spatial interval around the zero with full-width halfmaximum (FWHM)

$$
d \approx \frac{\lambda}{2 n \sin \alpha \sqrt{1+a I_{\max } / I}}
$$

where $a>0$ is a parameter that takes into consideration the shape of the zero'29, for example, whether it is a zero point or a zero line. For $I_{\max }=0$, we have the diffraction limit, whereas for $I_{\max } \gg I_{\mathrm{s}}$, the state is sharply defined. If $I(x, t)$ is designed to switch the dye off, any fluorescence photon must originate from this sharply defined interval $d$ around the zero. If, conversely, $I(x, t)$ is designed to switch the dye on, no photon will originate from that region around the zero. As the square root of $I_{\max } / I_{\mathrm{s}}$ increases, $d$ decreases.

For simplicity, let us now assume the first case, wherein $I(x, t)$ is off-switching, confining the 'on' state in a subdiffraction-sized region of dimension $d$. Molecules or tiny objects at distance $<\lambda /(2 n \sin \alpha)$ can now be resolved by shifting the intensity zeros across them and registering the signal for each position in space. If they are further apart than $d$, the molecules cannot be simultaneously in the 'on' state and hence are registered separately. Conversely, 
Figure 2 | Fluorescence switching strategies for super-resolution image assembly. To resolve details that are closer than the diffraction limit $\lambda /(2 n$ $\sin \alpha)$, current far-field fluorescence nanoscopy schemes switch molecular fluorescence on and off so that adjacent features or molecules do not emit simultaneously. Switching means transferring the molecules from a fluorescent state (A) to a dark state (B), or vice versa. There are two strategies: (a) In the targeted mode, a spatial light intensity distribution $I(x, t)$ having a zero intensity point in space switches the molecules such that one of the states-here A-is confined to sub-diffraction dimensions $d$. For example, in STED microscopy the zero-intensity point is realized by a doughnut-shaped beam $I(x, t)$ for molecular de-excitation (upper left corner) switching off all molecules that are not located at the zero, thus sharply confining a region with diameter $d \ll \lambda /(2 n \sin \alpha)$ in which the molecules are on (in state A). The image is assembled by shifting the pattern $I(x, t)$ over the sample (scanning) and recording adjacent features sequentially in time. Several molecules can reside in the same sub-diffraction-sized region. To parallelize the recording procedure (lower right corner), $I(x, t)$ can also feature an array of zero lines or points with pitch $>\lambda /(2 n \sin \alpha)$ and implement camera recording. To super-resolve in all directions, the line pattern must be tilted and scanned an appropriate number of times. Bottom, comparison of confocal (Conf) and STED image of immunolabeled vimentin in a mammalian cell, after linear deconvolution. Imaged by R. Medda using the compact supercontinuum STED microscope described by D. Wildanger et al. Opt. Expr. 16, 9614 (2008). (b) In the stochastic switching mode, such as in PALM and STORM, individual molecules are switched on (to state A) randomly in space, emitting $m>$ 1 photons in a row, while the surrounding molecules remain in the dark

state. The distance between the 'on' molecules should be $>\lambda /(2 n \sin \alpha)$ to facilitate recognition of individual molecules. Their coordinates are gained by calculation of their centroids. In the variant GSDIM, the molecules are first switched off to B and then pop up spontaneously in A. Bottom, comparison of a conventional wide-field image with a GSDIM image of microtubules (green) and peroxisomes (red) in mammalian cells. Imaged by J. Fölling as described in J. Fölling et al. Nat. Methods 5, 943 (2008). Scale bars, $1 \mu \mathrm{m}$. if they are closer than $d$, the molecules are inseparable because they can emit in concert. The resolution is thus given by $d$, and the coordinates of each molecule or little object are acquired through the position of the zero, with uncertainty $d$. This is how STED, GSD and RESOLFT discern molecules on the nanoscale.

To give an example, in STED microscopy $I(x, t)$ is usually formed into a doughnut that switches the fluorophores off except at its very center. Joining this beam with a regularly focused excitation beam and translating it across the specimen switches molecules on and off, so that those at distance $>d$ fluoresce consecutively, whereas those closer than $d$ sum their signals. Hence STED overcomes the diffraction barrier by making sure that narrowly spaced molecules of the same kind are in different states; that is, either on or off. GSD microscopy ${ }^{13,30,31}$ uses the same strategy except that it switches the molecules off by 'shelving' them for a short time in the dark, metastable triplet state. The ability to tune the spatial dimension $d$ in which the molecules are in different states demonstrates that the limiting role of diffraction is truly overcome.

The same readout principle is applied in SPEM ${ }^{14} / \mathrm{SSIM}^{15}$, except that $I(x, t)$ is used to switch the fluorophores on, thus confining the 'off' state. SPEM/SSIM has also introduced the application of many line-shaped zeros ('structured illumination') and thus parallelized detection from many sample coordinates using a camera. Molecules that are simultaneously 'off' fall within the same region of FWHM $d$ or are further apart than the diffraction limit $\lambda /(2 n \sin \alpha)$. Because objects that are further apart than $\lambda /(2 n \sin \alpha)$ can readily be separated, they need no time-sequential observation and can be observed in paralle ${ }^{15,16}$. Therefore, it is conceptually unnecessary to scan a zero further than the diffraction limit. This parallelization strategy can be applied to any targeted readout modality, including STED microscopy. However, parallelized schemes are particularly attractive in conjunc- a
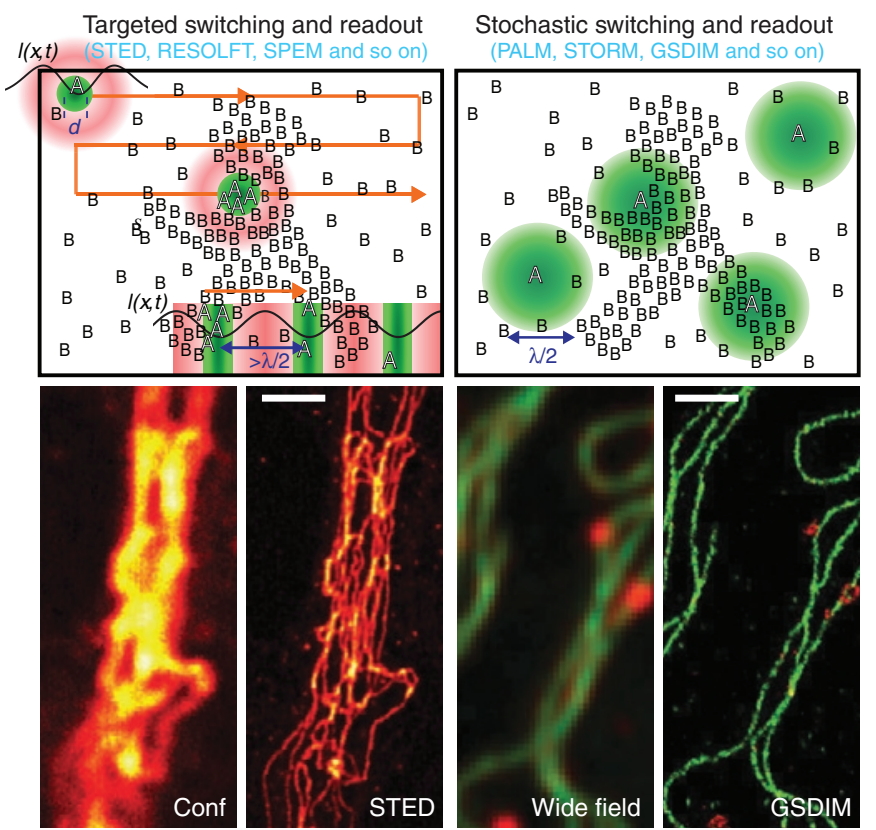

Stochastic switching and readout

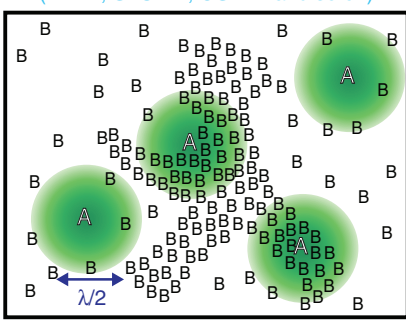


resentative number of calculated coordinates renders a number of calculated position tick marks that form an image.

Notably, some molecules may be localized more precisely than others, because $m$ varies from event to event following a statistical distribution. Therefore, to ensure a certain resolution, the stochastic readout mode defines a brightness threshold (for example, $m>M$ $\approx 50$ ), meaning that molecular events with smaller $m$ are discarded. $M$ will depend on the average number of photon emissions in the 'on' state and on the desired resolution, which, provided there is no background, exceeds $\lambda /\left[2 n(\sin \alpha) M^{1 / 2}\right]$. Thus PALM, STORM and other concepts using a stochastic readout have achieved remarkable resolution of $<20 \mathrm{~nm}$ in the focal plane $\mathrm{e}^{20,36,37}$.

To keep data acquisition short, the $m$ photons must be emitted quickly. A solution is to operate the camera at a fast frame rate $(\sim 500 \mathrm{~Hz})$ and apply an excitation intensity ensuring that the $m$ photons are emitted within the duration of a camera frame $(\sim 2 \mathrm{~ms}$ at present). The camera can be conveniently operated in asynchrony with the off-on-off switching cycle of the molecule, as put forth in the variant 'PALM with independently running acquisition' (PALMIRA) ${ }^{38,39}$ and GSDIM ${ }^{26}$. These concepts have also resorted to switching mechanisms, such as ground state depletion by dark state population, whereby both the (off-)switching and the production of the photon bunch can be realized with the same laser ${ }^{26,38-40}$. They probably are the simplest far-field nanoscopy systems to implement, because they require just uniform laser illumination, a freely running camera and appropriate software.

\section{Implementation considerations}

Simplicity of implementation is a major asset of PALM and STORM and of all nanoscopy modalities using stochastic molecular switching. However, the registration of the data and the correct image assembly require more caution ${ }^{41,42}$. In particular, one must avoid two molecules appearing simultaneously in the 'on' state within distance $\lambda /(2 n \sin \alpha)$ because they would be mistaken as a single, misplaced molecule. Therefore, when switching on (activating) molecules, the applied intensity must be adapted in inverse proportion to the local fluorophore concentration ${ }^{20}$. If $N$ gives the number of molecules within an area of diameter $\lambda /(2 n \sin \alpha)$, switching on with an intensity $<<I_{s} / N$ ensures that the 'on'-state molecules are far enough apart. Stronger intensities will lead to 'multiple molecule' events, whereas excessively weak intensities will unduly slow down the recording.

With STED microscopy, it is just the other way around. Setting up a STED system requires expertise in optics, but, once built, it can be used like a scanning (confocal) microscope. Observation of the labeling density is not needed because the resolution is physically determined by $d$.

There are also other differences between the two modalities. Whereas in PALM and STORM the $m>M$ photons from a single molecule are spent computing the molecule's coordinates, in STED all photons from all molecules within $d$ contribute to a pixel. Thus, it is possible to quickly discern tiny features of size $<d$ by fast scanning $^{43}$, as has been demonstrated by imaging synaptic vesicles in living neurons at video rate ${ }^{44}$. Moreover, because $d$ can be arbitrarily tuned in size, one can balance between high resolution and fast fluorescence collection, which is useful in many applications. Although it can deal with single molecules, the targeted readout mode does not rely on the detection of single molecules ${ }^{45-47}$, as does the stochastic readout mode; rather, it is an ensemble method that simultaneously records the signal of all molecules from the area targeted.

Yet targeting an area also means targeting 'empty' space, which, if there is a lot of it, slows the recording. Here, the stochastic mode has a clear advantage because it reads out only where molecules are present. Also, it discloses valuable information on a permolecule level ${ }^{48}$. The low background required for single-molecule readout operation explains why initial PALM and STORM recordings have mostly been implemented with total internal reflection schemes ${ }^{20,36}$, but, if background is low, three-dimensional recording is possible and has been demonstrated ${ }^{37,49}$. In contrast, STED microscopy can direct its nanosized spots to arbitrary positions inside a three-dimensional cell, as recently shown by imaging dendritic spines in living organotypic hippocampal slices ${ }^{50}$. This feature is also of great advantage when investigating fast (millisecond) dynamics of labeled molecules in nanosized areas in space by fluorescence fluctuation spectroscopy ${ }^{51}$, whereas an advantage of the stochastic readout, such as in PALM, is to track the movement of individual molecules in time ${ }^{25,41}$.

Aberrations affect both readout modes. In the targeted mode they may 'fill up' the zeros, impairing resolution and reducing signal. To render $d<30 \mathrm{~nm}$, the zeros should amount to less than $2 \%$ of $I_{\max }$. Likewise, aberrations distort the fluorescence spot that is required to precisely localize single molecules on a camera.

However, the most relevant practical difference between the two strategies is the economy of the switching ${ }^{19,27}$. In the targeted mode, the molecules are inevitably switched on and off several times, as the zeros are scanned across the sample. This is the price to be paid for the ability to grab the signal of many molecules residing in the same place all at the same time. In contrast, to be registered in the stochastic readout, a molecule needs to undergo only a single switching cycle (off $\rightarrow$ on $\rightarrow$ off). Unlike in STED, there is no need to switch off neighboring molecules in PALM and STORM because they are 'off' already. Unless the sample must be imaged repeatedly, the final dark state can be any dark (bleaching) state.

Unfortunately, available reversibly switchable fluorescent proteins and switchable organic fluorophores allow only a limited number of switching cycles. This is the reason why, although suggested ${ }^{16}$ for and demonstrated ${ }^{19,28,52}$ in the targeted mode RESOLFT, the low $I_{s}$ molecular switching transitions such as the (reversible) photoactivation of fluorescent proteins or organic fluorophores have been more effective in PALM and STORM. 'Switching fatigue' at present seems to be an obstacle to realizing nanoscopy based on targeted readout at ultralow illumination 19,28,52 . Conversely, 'switching fatigue' is to RESOLFT as discarding of events having $m<M$ photons is to PALM and STORM. The higher the required resolution, the more molecular events are discarded.

In any case, the future discovery of molecular switches affording many switching cycles will enable a far-field optical RESOLFT nanoscopy that can grab the fluorescence of several molecules within a physically predefined resolution range $d$. Such molecular switches should thus allow for highly parallelized, fast recording at intensities that are $10^{3}-10^{6}$ times lower than in STED microscopy. Similarly, the stochastic readout is expected to become substantially faster once faster cameras are available and $m$ can be increased substantially. A possible solution to increasing $m$ and its statistical distribution is to replace fluorescence switching with the switching of scattering events-for example, of colloidal 
particle pairs connected with a linker that changes its length upon photon absorption.

\section{The nonlinearity angle}

In the targeted mode, 'switching' actually means that a molecule is transferred from one state to the other with high probability. Because the probability of transition from one state to the other ideally depends exponentially on the applied intensity, one can readily interpret STED, GSD, SPEM/SSIM and RESOLFT as nonlinear optical concepts. (This nonlinearity, however, is fundamentally different from $m$-photon processes, in which the nonlinearity stems from the concomitant action of $m$ photons). By applying $I<<I_{s}$, the stochastic readout mode such as that used in PALM and STORM clearly avoids this nonlinear dependence on switching intensity. Still, there is an inherent nonlinear optical dependence in the stochastic mode as well, which, of course, is not based on the intensity used for switching, but on the intensity of molecular emission.

The reason for that is very simple. To be registered, a switchedon molecule has to send $m>M$ photons to the detector. Similar to $m$-photon excitation, in which $m$ photons need to arrive at the same molecule to produce a single excitation event, we require the same molecule to yield $>m$ detectable photons to produce a single detection event. Such an ' $m$-photon detection' event ${ }^{53}$ inherently depends nonlinearly (with order $m$ ) on the emitted fluorescence intensity. So, whereas a targeted readout mode such as STED microscopy uses a nonlinear dependence on the number of switching photons to actively define the coordinates of registration, the stochastic readout modes in PALM and STORM use a nonlinear dependence on the number of detected photons to find out the coordinates of registration.

Intimately connected with this aspect is that STED microscopy displays the measured photons linearly, whereas PALM and STORM represent the measured photons nonlinearly in the image. In the latter, photon events $m<M$ are clipped, which is why single-molecule switching images tend to be very clear, pointillistic ${ }^{54,55}$ and free of background. This is not to say that a nonlinear photon representation is unattractive, because, ultimately, we want to see the objects and not the photons. Producing images just 'by physics', STED, GSD and RESOLFT microscopy have the advantage of yielding linear photon representations. Nonetheless, applying deconvolution algorithms resulting in nonlinear representations of the measured photons can be very useful. In fact, the more the spot size $d$ approaches the size of the features, the more effective these algorithms become simply because there are fewer objects to be disentangled. In a sense, one may even argue that the localization in PALM and STORM is a perfected nonlinear deconvolution that uses the a priori assumption of having recorded a single molecule. In any case, attributing the super-resolution capabilities of STED, GSD, SSIM and related concepts to 'optical nonlinearities' but those of PALM and STORM to 'localization' misses the nonlinear aspects of the latter.

A nonlinearity aficionado may therefore argue that some sort of nonlinearity is needed after all. But then again, multiphoton absorption $^{56}$ and scattering ${ }^{57}$, which have been most popular in microscopy for almost two decades, are not effective in pushing the far-field optical resolution to the nanoscale ${ }^{58,59}$. In my view, the nonlinearity angle does not single out the central point of the current nanoscopy development, which is the saturated transition or switching between two states.

Similarly, localization per se cannot provide super resolution. This is why, although it had been known and used for a long time ${ }^{33,34}$, and routinely applied to single molecules ${ }^{60}$, localization alone did not provide nanoscale images ${ }^{61,62}$ with useful fluorophores and under ambient conditions ${ }^{63}$. The seminal transition from localization to imaging ${ }^{54,64}$, which has culminated in the PALM and STORM concept ${ }^{20-22}$, was enabled by fluorescence switching. This is the essence of all nanoscopy concepts discussed herein and the singular element without which none of them could produce an image.

\section{How we got here}

This brings us to the question of why the far-field diffraction barrier persisted for so long. A clue is to be found on Abbe's memorial (Fig. 1). Abbe's and Rayleigh's equations are about propagating waves, and, in fact, Abbe's theory is just about wave decomposition and the limited passband of spatial frequencies carried by waves through a microscope. In my view, the success of Abbe's theory directed too much attention on waves and on the passband. "A meaningful increase in resolution is not possible because of the limited passband" was for a long time a popular reason to dismiss any hope for better resolution with conventional objective lenses. Clearly, with conventional lenses ${ }^{65}$ there is still no way to change the propagation of waves such that the microscope's passband of wave spectra is expanded or the light more sharply confined than allowed by diffraction. Therefore, in the quest for nanoscale optical resolution, it seemed natural to trade off lenses and free wave propagation for mechanical confinement by nanosized tips, which is the philosophy of near-field optics ${ }^{66-69}$.

Of course, ideas were put forward that claimed to allow for 'farfield' super resolution, such as the Toraldo pupil filters ${ }^{70}$, the Lukosz principle $^{71}$ and, to some extent, also confocal microscopy ${ }^{72,73}$ but even under optimistic assessments these concepts could never have provided an improvement of more than a factor of two. Other considerations of super resolution ${ }^{74}$ were based on inadequate assumptions about the nature of diffraction and hence did not lead to valid conclusions. Sophisticated mathematical approaches of image restoration ${ }^{75}$ were successfully applied but were limited by the signalto-noise ratio prevalent in fluorescence images. The notion that optical nonlinearities could potentially improve the resolution also was around, but the popular multiphoton processes were clearly unsuitable, and so no one could specify how it could really be done. It remained a vague notion. For all these reasons, in the early 1990's, it was widely accepted that overcoming the diffraction barrier in a focusing light microscope was not realistic. The only effective pathway of optical microscopy to the nanoscale seemed to be given by the physical confinement of light, that is, by near-field optics ${ }^{66-69}$.

By placing the fluorophore-specifically, transitions between a dark and a bright state-at center stage $\mathrm{e}^{11,13,76}$, STED and GSD microscopy followed a radically different pathway. Instead of confining or isolating the light, $I(x, t)$, as near-field optics does, these concepts directly confined or isolated a molecular statespecifically, the fluorescent state-by transiently switching neighboring molecules into a dark state. This basic change in strategy is also reflected in the point-spread functions (PSFs) of STED and GSD microscopes. Whereas the PSFs of the standard far-field optical microscopes of that time basically describe diffractionlimited light intensity distributions (or higher orders thereof), the PSFs of STED and GSD microscopes mirror the spatial distribution of the fluorophore on-state as resulting from the deliberately imposed molecular switching ${ }^{11,13}$. The addition of related 
on-off transitions ${ }^{14,16-18}$, such as cis-trans isomerization and optical bistabilities, the parallelization for sequential large-area recording (SPEM/SSIM $)^{14,15}$, and the advent of stochastic single molecule switching followed by mathematical localization (PALM and STORM $)^{20-22}$ are other crucial milestones in far-field optical nanoscopy. Finally, it should be noted that it would have been impossible for the three aforementioned preeminent scientists of the nineteenth century to have considered such pathways because the concept of molecular states and state transitions required the confirmation of the existence of molecules and the advent of quantum mechanics.

\section{Where we are going}

Switching on and off is very effective for overcoming diffraction, but keeping many molecules off slows down the imaging process. Resorting to many distinguishable bright states with interstate transitions differing in the excitation and emission wavelengths will be a powerful extension because it is like placing many differently colored stickers on the tiny little objects in the sample. Such methods should greatly speed up the process and help in reducing the acquisition time substantially.

All nanoscopy methods can provide improved axial resolution $^{12,49,77-79}$, but with a single lens the resolution along the optical axis always remains worse than its focal plane counterpart. The coherent use of two opposing lenses as pioneered in 4Pi microscopy and $\mathrm{I}^{5} \mathrm{M}$ will, however, facilitate an axial resolution improvement by up to sevenfold with these switch-based nanoscopy methods. In fact, this was demonstrated with STED a few years ago $^{77,80}$ and, most recently, led to a method for isotropic nanoscale three-dimensional resolution called isoSTED ${ }^{81}$. A similar gain has been predicted for the stochastic single-molecule switching modalities $^{82}$.

Although it has been shown for more than a decade that a powerful key to nanoscale resolution is the imaged dye itself $11,13,16,17,76$, the recent, overwhelming success of nanoscopy imaging has made this notion abundantly obvious. The designing of molecular markers specifically for outperforming the diffraction barrier ${ }^{16,17}$ will be highly rewarding. For the stochastic single molecule switching modalities, these will be molecules that burst out large $m$ in the shortest possible period of time, whereas for the targeted switching modalities the goal should be molecules that can undergo many on-off cycles. With the right marker molecules to hand, emerging optical nanoscopy is bound to transform the life sciences.

Notably, in the targeted readout mode, the 'on' state need not be fluorescent; in fact it need not even be an optical emitter, just detectable ${ }^{17}$. This is because the coordinates are defined by the zero, and that is enough. In the stochastic switching mode it is just the other way around: the 'on' state need not be activated by light ${ }^{26}$. The only requirement with regard to optics is that it must emit $m$ photons that can be detected by a camera-like detector.

So if we had the perfect marker for the stochastic single-molecule switching based readout, what would we obtain? Clearly, we would obtain the position of a molecule with a precision of a fraction of a nanometer. Although this information would be invaluable for mapping the sample, it would not tell us much about the molecule itself.

The situation is different, though, in a purely physics-based design such as STED, in which the molecule is actively driven between two states at defined spatial coordinates. Equation (1) is provocative because $I_{\max } / I_{\mathrm{s}} \rightarrow \infty$ yields $d \rightarrow 0$, thus raising the question as to what happens if $I_{\max }$ is increased to very high values. Let us imagine a molecule of $0.3-3 \mathrm{~nm}$ in size placed at the point of zero intensity of the electric field. If we steadily increase $I_{\max }$, the light becomes non-negligible at the periphery of the electron orbitals while their central part is still being weakly exposed. Because different parts of the orbitals experience electric fields of different strengths, they will have differently pronounced contributions to a light-induced transition. The spatial structure of the light field cannot be ignored with respect to the size of the molecules, and the dipole approximation is not expected to remain valid. Translating the zero across the molecule changes the transition probability of, say, excitation, stimulated emission, photo conversion or photoisomerization as a function of the orbital structure and the role of certain parts in certain transitions. No matter how quickly this 'inframolecular' photophysics or photochemistry becomes reality, this fascinating prospect once more highlights there is plenty to discover down at the bottom-with lenses and focused light!

\section{ACKNOWLEDGMENTS}

I thank E. Rittweger and B. Rankin for help in preparing the figures and various suggestions for improving the presentation. Critical reading by A. Schönle, and also by S. Jakobs, C. Eggeling and J. Jethwa, is gratefully acknowledged.

\section{Published online at http://www.nature.com/naturemethods/ Reprints and permissions information is available online at http://npg. nature.com/reprintsandpermissions/}

1. Verdet, É. Leçons d'optique physique (Victor Masson et fils, Paris, 1869).

2. Abbe, E. Beiträge zur Theorie des Mikroskops und der mikroskopischen Wahrnehmung. Arch. Mikr. Anat. 9, 413-468 (1873).

3. Lord Rayleigh. On the theory of optical images, with special reference to the microscope. Philos. Mag. XLII, 167-195 (1896).

4. Hell, S.W. Double confocal microscope. European Patent 0491289 (1990).

5. Hell, S.W. \& Stelzer, E.H.K. Fundamental improvement of resolution with a $4 \mathrm{Pi}$-confocal fluorescence microscope using two-photon excitation. Opt. Commun. 93, 277-282 (1992).

6. Schrader, M. \& Hell, S.W. 4Pi-confocal images with axial superresolution. J. Microsc. 183, 189-193 (1996).

7. Hell, S.W., Schrader, M. \& van der Voort, H.T.M. Far-field fluorescence microscopy with three-dimensional resolution in the $100 \mathrm{~nm}$ range. J. Microsc. 185, 1-5 (1997).

8. Gustafsson, M.G.L., Agard, D.A. \& Sedat, J.W. Sevenfold improvement of axial resolution in 3D widefield microscopy using two objective lenses. Proc. SPIE 2412, 147-156 (1995).

9. Gustafsson, M.G.L., Agard, D.A. \& Sedat, J.W. I $^{5}$ M: 3D widefield light microscopy with better than $100 \mathrm{~nm}$ axial resolution. J. Microsc. 195, 10-16 (1999).

10. Burns, D.H., Callis, G.D., Christian, G.D. \& Davidson, E.R. Strategies for attaining superresolution using spectroscopic data as constraints. Appl. Opt. 24, 154-160 (1985).

11. Hell, S.W. \& Wichmann, J. Breaking the diffraction resolution limit by stimulated emission: stimulated emission depletion microscopy. Opt. Lett. 19, 780-782 (1994).

12. Klar, T.A., Jakobs, S., Dyba, M., Egner, A. \& Hell, S.W. Fluorescence microscopy with diffraction resolution limit broken by stimulated emission. Proc. Natl. Acad. Sci. USA 97, 8206-8210 (2000).

13. Hell, S.W. \& Kroug, M. Ground-state depletion fluorescence microscopy, a concept for breaking the diffraction resolution limit. Appl. Phys. B 60, 495-497 (1995)

14. Heintzmann, R., Jovin, T.M. \& Cremer, C. Saturated patterned excitation microscopy - a concept for optical resolution improvement. J. Opt. Soc. Am. A 19, 1599-1609 (2002).

15. Gustafsson, M.G.L. Nonlinear structured-illumination microscopy: wide-field fluorescence imaging with theoretically unlimited resolution. Proc. Natl. Acad. Sci. USA 102, 13081-13086 (2005).

16. Hell, S.W. Toward fluorescence nanoscopy. Nat. Biotechnol. 21, 1347-1355 (2003).

17. Hell, S.W. Strategy for far-field optical imaging and writing without 
diffraction limit. Phys. Lett. A 326, 140-145 (2004).

18. Hell, S.W., Dyba, M. \& Jakobs, S. Concepts for nanoscale resolution in fluorescence microscopy. Curr. Opin. Neurobiol. 14, 599-609 (2004).

19. Hofmann, M., Eggeling, C., Jakobs, S. \& Hell, S.W. Breaking the diffraction barrier in fluorescence microscopy at low light intensities by using reversibly photoswitchable proteins. Proc. Natl. Acad. Sci. USA 102, 17565-17569 (2005).

20. Betzig, E. et al. Imaging intracellular fluorescent proteins at nanometer resolution. Science 313, 1642-1645 (2006).

21. Rust, M.J., Bates, M. \& Zhuang, X. Sub-diffraction-limit imaging by stochastic optical reconstruction microscopy (STORM). Nat. Methods 3, 793-796 (2006).

22. Hess, S.T., Girirajan, T.P.K. \& Mason, M.D. Ultra-high resolution imaging by fluorescence photoactivation localization microscopy. Biophys. J. 91, 4258-4272 (2006).

23. Sharonov, A. \& Hochstrasser, R.M. Wide-field subdiffraction imaging by accumulated binding of diffusing probes. Proc. Natl. Acad. Sci. USA 103, 18911-18916 (2006).

24. Heilemann, M. et al. Subdiffraction-resolution fluorescence imaging with conventional fluorescent probes. Angew. Chem. 47, 6172-6176 (2008).

25. Biteen, J.S. et al. Super-resolution imaging in live Caulobacter crescentus cells using photoswitchable EYFP. Nat. Methods 5, 947-949 (2008).

26. Fölling, J. et al. Fluorescence nanoscopy by ground-state depletion and single-molecule return. Nat. Methods 5, 943-945 (2008).

27. Hell, S.W. Far-field optical nanoscopy. Science 316, 1153-1158 (2007).

28. Schwentker, M.A. et al. Wide-field subdiffraction RESOLFT microscopy using fluorescent protein photoswitching. Microsc. Res. Tech. 70, 269-280 (2007).

29. Harke, B. et al. Resolution scaling in STED microscopy. Opt. Express 16, 4154-4162 (2008).

30. Hell, S.W. Increasing the resolution in fluorescence light microscopy by point-spread-function engineering. in Topics in Fluorescence Spectroscopy (ed. Lakowicz, J.R.) 361-422 (Plenum, New York, 1997).

31. Bretschneider, S., Eggeling, C. \& Hell, S.W. Breaking the diffraction barrier in fluorescence microscopy by optical shelving. Phys. Rev. Lett. 98, 218103 (2007).

32. Hell, S.W. \& Schönle, A. Nanoscale resolution in far-field fluorescence microscopy. in Science of Microscopy (eds., Hawkes, P.W. and Spence, J.C.H) 790-834 (Springer, New York, 2007).

33. Heisenberg, W. The Physical Principles of the Quantum Theory (Chicago Univ. Press, Chicago, 1930)

34. Bobroff, N. Position measurement with a resolution and noise-limited instrument. Rev. Sci. Instrum. 57, 1152-1157 (1986).

35. Betzig, E. Proposed method for molecular optical imaging. Opt. Lett. 20, 237-239 (1995).

36. Bates, M., Huang, B., Dempsey, G.P. \& Zhuang, X. Multicolor super-resolution imaging with photo-switchable fluorescent probes. Science 317, 1749-1753 (2007).

37. Fölling, J. et al. Photochromic rhodamines provide nanoscopy with optical sectioning. Angew. Chem. Int. Ed. 46, 6266-6270 (2007).

38. Egner, A. et al. Fluorescence nanoscopy in whole cells by asnychronous localization of photoswitching emitters. Biophys. J. 93, 3285-3290 (2007).

39. Geisler, C. et al. Resolution of $\lambda / 10$ in fluorescence microscopy using fast single molecule photo-switching. Appl. Phys. A Mater. Sci. Process. 88, 223-226 (2007).

40. Steinhauer, C., Forthmann, C., Vogelsang, J. \& Tinnefeld, P. Superresolution microscopy on the basis of engineered dark states. J. Am. Chem. Soc. 10.1021/ja806590m (2008).

41. Shroff, H., Galbraith, C.G., Galbraith, J.A. \& Betzig, E. Live-cell photoactivated localization microscopy of nanoscale adhesion dynamics. Nat. Methods 5, 417-423 (2008).

42. Shroff, H. et al. Dual-color superresolution imaging of genetically expressed probes within individual adhesion complexes. Proc. Natl. Acad. Sci. USA 104, 20308-20313 (2007).

43. Westphal, V., Lauterbach, M.A., Di Nicola, A. \& Hell, S.W. Dynamic far-field fluorescence nanoscopy. New J. Phys. 9, 435 (2007).

44. Westphal, V. et al. Video-rate far-field optical nanoscopy dissects synaptic vesicle movement. Science 320, 246-249 (2008).

45. Moerner, W.E. \& Kador, L. Optical detection and spectroscopy of single molecules in a solid. Phys. Rev. Lett. 62, 2535-2538 (1989).

46. Orrit, M. \& Bernard, J. Single pentacene molecules detected by fluorescence excitation in a p-terphenyl crystal. Phys. Rev. Lett. 65, 2716-2719 (1990).

47. Moerner, W.E. Single-molecule mountains yield nanoscale cell images. Nat. Methods 3, 781-782 (2006).

48. Schönle, A. \& Hell, S.W. Fluorescence nanoscopy goes multicolor. Nat. Biotechnol. 25, 1234-1235 (2007).

49. Huang, B., Wang, W., Bates, M. \& Zhuang, X. Three-dimensional super- resolution imaging by stochastic optical reconstruction microscopy. Science 319, 810-813 (2008)

50. Nägerl, V.U., Willig, K.I., Hein, B., Hell, S.W. \& Bonhoeffer, T. Live-cell imaging of dendritic spines by STED microscopy. Proc. Natl. Acad. Sci. USA 105, 18982-18987 (2008).

51. Kastrup, L., Blom, H., Eggeling, C. \& Hell, S.W. Fluorescence fluctuation spectroscopy in subdiffraction focal volumes. Phys. Rev. Lett. 94, 178104 (2005).

52. Dedecker, P. et al. Subdiffraction imaging through the selective donut-mode depletion of thermally stable photoswitchable fluorophores: numerical analysis and application to the fluorescent protein Dronpa. J. Am. Chem. Soc. 129, 16132-16141 (2007).

53. Hell, S.W., Soukka, J. \& Hänninen, P.E. Two- and multiphoton detection as an imaging mode and means of increasing the resolution in far-field light microscopy. Bioimaging 3, 64-69 (1995).

54. Lidke, K.A., Rieger, B., Jovin, T.M. \& Heintzmann, R. Superresolution by localization of quantum dots using blinking statistics. Opt. Express 13, 7052-7062 (2005).

55. Heintzmann, R. \& Ficz, G. Breaking the resolution limit in light microscopy. Brief. Funct. Genomic. Proteomic. 5, 289-301 (2006).

56. Denk, W., Strickler, J.H. \& Webb, W.W. Two-photon laser scanning fluorescence microscopy. Science 248, 73-76 (1990).

57. Zumbusch, A., Holtom, G.R. \& Xie, X.S. Three-dimensional vibrational imaging by coherent anti-stokes Raman scattering. Phys. Rev. Lett. 82, 4142-4145 (1999).

58. Schönle, A., Hänninen, P.E. \& Hell, S.W. Nonlinear fluorescence through intermolecular energy transfer and resolution increase in fluorescence microscopy. Ann. Phys. 8, 115-133 (1999).

59. Schönle, A. \& Hell, S.W. Far-field fluorescence microscopy with repetetive excitation. Eur. Phys. J. D 6, 283-290 (1999).

60. Weiss, S. Fluorescence spectroscopy of single biomolecules. Science 283, 1676-1683 (1999).

61. Bornfleth, H., Satzler, K., Eils, R. \& Cremer, C. High-precision distance measurements and volume-conserving segmentation of objects near and below the resolution limit in three-dimensional confocal fluorescence microscopy. J. Microsc. 189, 118-136 (1998).

62. Hildenbrand, G. et al. Nano-sizing of specific gene domains in intact human cell nuclei by Spatially Modulated Illumination (SMI) light microscopy. Biophys. J. 88, 4312-4318 (2005).

63. van Oijen, A.M., Köhler, J., Schmidt, J., Müller, M. \& Brakenhoff, G.J. Farfield fluorescence microscopy beyond the diffraction limit. J. Opt. Soc. Am. A 16, 909-915 (1999).

64. Qu, X., Wu, D., Mets, L. \& Scherer, N.F. Nanometer-localized multiple singlemolecule fluorescence microscopy. Proc. Natl. Acad. Sci. USA 101, 1129811303 (2004)

65. Pendry, J.B. Negative refraction makes a perfect lens. Phys. Rev. Lett. 85, 3966-3969 (2000).

66. Synge, E.H. A suggested method for extending microscopic resolution into the ultra-microscopic region. Philos. Mag. 6, 356 (1928).

67. Pohl, D.W., Denk, W. \& Lanz, M. Optical stethoscopy: image recording with resolution $\lambda / 20$. Appl. Phys. Lett. 44, 651-653 (1984).

68. Lewis, A., Isaacson, M., Harootunian, A. \& Murray, A. Development of a 500 Å resolution light microscope. Ultramicroscopy 13, 227-231 (1984).

69. Betzig, E., Chichester, R.J., Lanni, F. \& Taylor, D.L. Near-field fluorescence imaging of cytoskeletal actin. Bioimaging 1, 129-136 (1993).

70. Toraldo di Francia, G. Supergain antennas and optical resolving power. Nuovo Cimento 9 Suppl., 426-435 (1952).

71. Lukosz, W. Optical systems with resolving powers exceeding the classical limit. J. Opt. Soc. Am. 56, 1463-1472 (1966).

72. Minsky, M. Microscopy apparatus. US patent 3,013,467 (1961).

73. Sheppard, C.J.R. \& Wilson, T. The theory of scanning microscopes with Gaussian pupil functions. J. Microsc. 114, 179-197 (1978).

74. Cremer, C. \& Cremer, T. Considerations on a laser-scanning-microscope with high resolution and depth of field. Microsc. Acta 81, 31-44 (1978).

75. Carrington, W.A. et al. Superresolution in three-dimensional images of fluorescence in cells with minimal light exposure. Science 268, 1483-1487 (1995).

76. Hell, S.W. Improvement of lateral resolution in far-field light microscopy using two-photon excitation with offset beams. Opt. Commun. 106, 19-24 (1994).

77. Dyba, M. \& Hell, S.W. Focal spots of size $\lambda / 23$ open up far-field fluorescence microscopy at $33 \mathrm{~nm}$ axial resolution. Phys. Rev. Lett. 88, 163901 (2002).

78. Fölling, J. et al. Fluorescence nanoscopy with optical sectioning by two-photon induced molecular switching using continuous-wave lasers. ChemPhysChem 9, 321-326 (2008). 
79. Juette, M.F. et al. Three-dimensional sub-100 nm resolution fluorescence microscopy of thick samples. Nat. Methods 5, 527-529 (2008).

80. Dyba, M., Jakobs, S. \& Hell, S.W. Immunofluorescence stimulated emission depletion microscopy. Nat. Biotechnol. 21, 1303-1304 (2003).

81. Schmidt, R. et al. Spherical nanosized spot unravel the interior of cells. Nat. Methods 4, 81-86 (2008).

82. v Middendorff, C., Egner, A., Geisler, C., Hell, S.W. \& Schönle, A. Isotropic 3D Nanoscopy based on single emitter switching. Opt. Express 16, 20774-20788 (2008).

83. Nagorni, M. \& Hell, S.W. 4Pi-confocal microscopy provides three-dimensional images of the microtubule network with 100 - to $150-\mathrm{nm}$ resolution. J. Struct. Biol. 123, 236-247 (1998).

84. Egner, A. \& Hell, S.W. Fluorescence microscopy with super-resolved optical sections. Trends Cell Biol. 15, 207-215 (2005).

85. Shao, L. et al. $\mathrm{I}^{5} \mathrm{~S}$ : wide-field light microscopy with $100-\mathrm{nm}$-scale resolution in three dimensions. Biophys. J. 94, 4971-4983 (2008).

86. Willig, K.I., Rizzoli, S.O., Westphal, V., Jahn, R. \& Hell, S.W. STED-microscopy reveals that synaptotagmin remains clustered after synaptic vesicle exocytosis. Nature 440, 935-939 (2006).

87. Hell, S.W. et al. Nanoscale resolution with focused light: stimulated emission depletion and other reversible saturable optical fluorescence transitions microscopy concepts. in Handbook of Biological Confocal Microscopy (ed. Pawley, J.) 571-579 (Springer, New York, 2006).

88. Hell, S.W., Jakobs, S. \& Kastrup, L. Imaging and writing at the nanoscale with focused visible light through saturable optical transitions. Appl. Phys. A 77, 859-860 (2003). 